\title{
Reflexiones sobre la idea de América Latina y sus contribuciones a las terapias ocupacionales del sur
}

\author{
Reflexóes sobre a ideia de América Latina e suas contribuiçóes às terapias \\ ocupacionais do sul
}

\section{Reflections on the idea of Latin America and its contributions to southern occupational therapies}

\author{
Mónica Matilde Díaz-Leiva ${ }^{\mathrm{a}, \mathrm{b}, \mathrm{c}}$ (D), Ana Paula Serrata Malfitano ${ }^{\mathrm{c}}$ \\ ${ }^{a}$ Universidad de Santiago de Chile - USACH, Santiago, Chile.

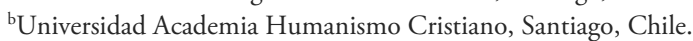 \\ 'Universidade Federal de São Carlos - UFSCar, São Carlos, SP, Brasil.
}

Cómo citar: Díaz-Leiva, M. M., \& Malfitano, A. P. S. (2021). Reflexiones sobre la idea de América Latina y sus contribuciones a las terapias ocupacionales del sur. Cadernos Brasileiros de Terapia

Ocupacional, 29, e2568. https://doi.org/10.1590/2526-8910.ctoEN1961

\begin{abstract}
Resumen
Se presenta una reflexión sobre las narrativas que se han elaborado en torno a América Latina y desde las cuales han emergido una serie de categorías que posibilitan determinadas y diferentes lecturas sobre nuestro continente. El objetivo es analizar estas narrativas e identificar lo que nos ofrecen para contribuir al debate sobre las Terapias Ocupacionales del Sur considerando que su despliegue tiene directa relación con las discusiones que, desde hace varias décadas, se vienen produciendo en nuestra región. Para ello se realiza un ensayo reflexivo en torno a teorías sociales que incluyen narrativas anticoloniales, poscoloniales, estudios subalternos, decoloniales, entre otras, seleccionando cuatro asuntos que contribuyen a la reflexión de las Terapias Ocupacionales del Sur: la heterogeneidad contradictoria de América Latina, la colonialidad como legado trans-generacional de la colonización, la politica de representación que ha asumido el mundo letrado o intelectual para observar, estudiar y representar la "alteridad" y la colonialidad interna como punto de arranque de procesos de descolonización. Se concluye que las terapias ocupacionales del sur se han configurado como un lugar de habla y reconocimiento de las narrativas y prácticas locales-regionales, teniendo una gran potencialidad para movilizar las relaciones y asimetrías de poder tanto a nivel local como global. La idea de América Latina nos permite identificar las maneras en que se ha transformado el curso de la profesión y se siguen produciendo nuevos sentidos y prácticas en respuesta a los contextos históricos y temporalidades en este continente.
\end{abstract}

Palabras-clave: Terapia Ocupacional, América Latina, Diversidad Cultural. 


\section{$\underline{\text { Resumo }}$}

Apresenta-se uma reflexão sobre as narrativas que foram elaboradas em torno do que é a América Latina e das quais emergiram uma série de categorias que permitem determinadas e diferentes leituras sobre o nosso continente. Objetiva-se analisar tais narrativas e identificar o que oferecemos para contribuir com o debate sobre as terapias ocupacionais do sul, considerando que a sua implantação atual está diretamente relacionada com as discussóes que, por décadas, vem ocorrendo em nossa regiáo. Para tanto, foi realizado um ensaio reflexivo em torno de teorias sociais, incluindo narrativas anticoloniais, pós-coloniais, estudos subalternos, descoloniais, entre outros, selecionando quatro questôes que contribuem para a reflexão das terapias ocupacionais do sul: a heterogeneidade contraditória da América Latina, a colonialidade como legado transgeracional da colonização, as politicas de representação que tem assumido o mundo intelectual para observar, estudar e representar a "alteridade" e a colonialidade interna como ponto de partida dos processos de descolonização. Conclui-se que as terapias ocupacionais do sul são reconhecidas pelas narrativas e práticas locais e regionais, tendo grande potencial para mobilizar relaçóes e assimetrias de poder local e globalmente. A ideia da América Latina nos permite identificar as formas pelas quais se tem transformado o curso da profissão e como seguem produzindo novos significados e práticas em resposta aos contextos históricos e temporalidades neste continente.

Palavras-chave: Terapia Ocupacional, América Latina, Diversidade Cultural.

\section{$\underline{\text { Abstract }}$}

It is presented a reflection about the narratives around Latin American concepts and the categories that emerged from these concepts, making the possibility of different readings about our continent. The objective of this article is analyzing these narratives and identifying what they offer to contribute to Southern Occupational Therapies debate. It is considered that their current deployment is directly related to the discussions about them, which have been taking place for several decades in our region. To this end, a reflexive essay is presented based on social theories, including anticolonial, postcolonial, subaltern, and decolonial studies, among others. We have chosen for debates to contribute to the reflection of Southern Occupational Therapies: the contradictory heterogeneity of Latin America, the coloniality as a transgenerational legacy of colonization, politics of representation in the intellectual world and how they have observed, studied, and represented "otherness" and the internal coloniality, as a starting point for processes of decolonization. It is concluded that the Southern Occupational Therapies have been recognized local-regional narratives and practices, having a great potential to mobilize power relations and asymmetries both locally and globally. The idea of Latin America allows us to identify how the course of the profession has been transformed and new meanings and practices have produced in response to historical contexts and temporalities in this continent.

Keywords: Occupational Therapy, Latin America, Cultural Diversity. 


\section{Introducción}

Dentro de las terapias ocupacionales críticas que se han venido produciendo, desde hace varias décadas, especialmente en Latinoamérica, las terapias ocupacionales del sur vienen a proponer un ejercicio reflexivo desde las condiciones específicas, diferentes y equivalentes, que concurren históricamente en lo que contemporáneamente configura Latinoamérica (Cottet, 2016). Esto es proponer una transformación de las relaciones sur-norte, en los diversos campos del saber y de la vida cotidiana, atravesados por largo tiempo por una concepción binaria que marcó una tendencia de concebir el norte como el productor de las ideas y el sur como su ejecutor (Richard, 1997).

Las Terapias Ocupacionales del sur nombran aquellas prácticas localizadas que han surgido desde diversas comunidades de terapeutas ocupacionales que, desde Latinoamérica y del sur global, ponen en movimiento una capacidad de hacer memoria y pensarse a sí mismas en relación con sus prácticas concretas y conocimientos producidos (Oliver, 1990; Barros, 1991; Guajardo, 1992, 2013, 2016; Galheigo, 2003; Herrera \& Valderrama, 2013; Zorzoli et al., 2014; Duarte et al., 2016; Testa et al., 2016). Recordemos que hablar de Terapias Ocupacionales del Sur ${ }^{1}$ no hace alusión exclusivamente al territorio geográfico ni solamente a América del Sur, sino que corresponde a un sur epistémico, un sur que comparte las huellas del colonialismo en sus formas de vida y por ello, desde esa experiencia compartida, ha podido construir un proyecto que reconoce otros principios políticos, éticos, económicos y epistémicos, distintos a los que fueron impuestos, desde el discurso logocentrista, en diversos continentes y en nuestra disciplina.

Las terapias ocupacionales del sur serían una plataforma móvil que pone en primer plano las asimetrías de poder y sus efectos en las subjetividades tanto de quienes se encuentran en el lugar de la disciplina como de quienes se encuentran en el lugar de su intervención o estudio. Se proponen activar la capacidad de autocrítica como una posicionalidad atenta frente a las diversas formas de reproducción del mismo orden que critica, por cuanto las huellas de la colonización se caracterizan por su capacidad de infiltración, tanto en los que experimentaron la negación de sus historias ${ }^{2}$ como en quienes la impusieron ${ }^{3}$. Esto no significa aislarse de la discusión global ni mucho menos desconocer el valor de las producciones en otros continentes, eso sería una contradicción, se trata de poner en común una reflexión crítica desde el sur global/latinoamericano, bajo el formato de una invitación a pensar desde la propia colonialidad interna que encarnamos (Cusicanqui, 2010).

En cuanto a la idea de América Latina, este articulo pretende aportar con diversas perspectivas que, muchas veces, son tratadas como si fuesen similares, no obstante, tal como lo señalan las teorías sociales que discuten estos diversos enfoques, cada narrativa debe ser analizada, porque es constitutiva y evaluativa en tanto informa y ayuda a definir las prácticas que describe, yendo más allá de una mera distinción terminológica (Benhabib, 2006). Así, categorías como anticolonialismo, latinomericanismo, postcolonialismo, decolonialismo, estudios subalternos, entre otros, abordan distintas

\footnotetext{
${ }^{1}$ Se puede localizar en distintos puntos geográficos que comparten el mismo lugar de enunciación, como América Central, África, Asia y Europa. Así mismo, habitar el sur geográfico, no es sinónimo de prácticas al sur de la terapia ocupacional.

${ }^{2}$ Nos referimos principalmente a Suramérica: México, América Central, el Caribe y América del Sur, Asia y África.

${ }^{3}$ Nos referimos a Europa y Estados Unidos principalmente.
} 
producciones del saber académico, algunas de las cuales, pueden crear una imagen esencialista de América Latina y discursivamente un ámbito de "marginalidad" y de "exterioridad", considerando las "fuerzas que experimentaban en ese momento las instituciones productoras del saber" (Castro-Gómez, 1998, p. 3). De allí la importancia de hacer una revisión abreviada de cuatro asuntos que surgen de estas diversas perspectivas y que nos parecen disparadores de una cadena de reflexiones sobre América Latina y su relación con las Terapias Ocupacionales del Sur. El primer asunto es la heterogeneidad contradictoria de América Latina, por sobre la idea de homogeneidad identitaria, el segundo es la colonialidad como legado trans-generacional de la colonización, el tercer asunto es la politica de representación que ha asumido el mundo letrado o intelectual para observar, estudiar y representar a la "alteridad" y finalmente, el asunto de la colonialidad interna.

\section{Primer asunto: sobre la heterogeneidad contradictoria de América Latina}

Hablar sobre América, es en sí mismo un concepto heterogéneo, polisémico y en su origen eurocéntrico, propio de un logos o razón universal e instrumental ${ }^{4}$, principal productor de la historia de América Latina. La invención del concepto "América Latina" en la historia universal, irrumpe y se crea durante la colonización del territorio de Tawantisuyo, Anahuac y Abya Yala, según la llamaban los pueblos que la habitaban hasta ese momento.

Antes de 1492, América no figuraba en ningún mapa, ni siquiera en el de los pueblos que vivían en el valle de Anahuac (territorio azteca) y Tawantinsuyu (territorio inca). Los españoles y los portugueses, únicos ocupantes europeos durante el siglo XVI, bautizaron el continente cuyo control y posesión estaba en sus manos. [...] El territorio existía y los pobladores también, por supuesto, pero ellos daban su propio nombre al lugar donde vivían (Mignolo, 2007, p. 28).

Para los colonizadores, se trataba de pueblos sin historia, de territorios nuevos, exóticos, y extraños, de seres que no alcanzaban el estatus de seres humanos, estaban fuera de la historia, por lo cual debían ser civilizados y evangelizados por el mundo letrado. La idea de América Latina fue construida fundamentalmente desde la lectura de Occidente, lectura producida a partir de un discurso colonialista de esta parte del mundo "[...] después de todo, el continente americano existe solo como una consecuencia de la expansión colonial europea y los relatos de esa expansión desde el punto de vista europeo, es decir, la perspectiva de la modernidad [...]" (Mignolo, 2005, p. 16). No existe una sola historia sobre este continente, al contrario, podría ser contada de diversas formas, aunque la versión eurocéntrica y moderna es la que ha ocupado una posición hegemónica y la que se ha legitimado incluso en las Ciencias Sociales y en diversos campos del saber. Otras versiones, siempre han existido, pero de modo poco visible, acalladas por el poder del logos universal, no obstante, se han resistido a modelos

\footnotetext{
${ }^{4}$ En contraposición a una razón situada cuyos límites siempre son desbordados por los contextos y prácticas que pretende explicar o comprender.
} 
universales por medio de prácticas y narrativas anti-coloniales, de(s)coloniales o giros decoloniales.

Algunas narrativas sobre América Latina, han intentado diversificar y proponer nuevos relatos, no obstante, otras han actuado como una máquina de homogeneización, que aun cuando parecieran promover y preservar la diferencia (Moreiras, 1996; CastroGómez, 1998), lo que en realidad hacen con las diferencias es controlarlas, homogenizarlas y ponerlas al servicio de una representación global administrada desde el “centro". Otras, en cambio, como señala Anzaldúa (1987) en su libro la nueva mestiza, proponen espacios intermedios y cruces fronterizos de orden epistémico cultural o como Cusicanqui (2010), quien plantea que América Latina está cruzada por una heterogeneidad contradictoria, derivada de la cultura $\mathrm{Ch}{ }^{\mathrm{ixi}}{ }^{5}$ en la región, lo cual significa que existe una yuxtaposición de ideas, creencias, prácticas que pueden coexistir y mantener una relación conflictiva. De cierta manera, asumen que no es posible unificar, ni pensar en una identidad latinoamericana, menos en creer que es posible una armonía idealizada, al contrario, el escenario latinoamericano ha estado marcado por una serie de luchas especialmente por el reconocimiento de la diferencia.

Finalmente los enfoques decoloniales y descoloniales vienen también a criticar fuertemente esos logos oficiales que clasifican y estratifican las historias otras en el lugar de la alteridad, otras inferiorizadas, desde el discurso colonialista-eurocéntrico creando una serie de esquemas binarios caracterizados por este valor asimétrico que se le otorga a la alteridad: Europa/América, colonizadores/colonizados, culto/popular, letra/oralidad, global/local, centro/periferia, civilizados/bárbaros, español o portugués/indígena, blanco/negro, hombre/mujer, quechua/español, entre otros. De ahí la importancia de recordar que esta fabricación eurocéntrica de la idea de América Latina surge sin referencia a la historia y contexto específico de este territorio y, por tanto, "sin ninguna referencia a un contexto de sentido" (Garcia, 2012, p.81).

\section{Segundo asunto: colonialidad como legado trans-generacional de la colonización.}

Diversos autores plantean el impacto de la colonización en América Latina, por supuesto con diferentes matices y tonalidades, por ejemplo, en Brasil, Florestan Fernandes (1973, p. 11) en su libro Capitalismo Dependiente y clases sociales en América Latina, señala "as naçóes latino-americanas são produtos da 'expansão da civilização ocidental', isto é, de um tipo moderno de colonialismo organizado e sistemático”. Por su parte, en Colombia, Fals Borda (2009, p. 408) señalaba que los procesos de colonización tuvieron el mismo corolario "esto puede comprobarse en Brasil, Colombia, Guatemala, Argentina, Perú, Ecuador y Chile”. En Perú, Quijano (1992) ha sido también un referente importante para las teorías sociales de América Latina, igual que Enrique Dussel (1997) filósofo argentino radicado en México por largos años, quien propuso una ética material de la vida para reconocer las vidas negadas de las víctimas de los sistemas de dominación colonial. De manera particular diversas autoras feministas, además de denunciar los efectos generales de la colonización, establecen que el colonialismo impuso fuertes procesos de dominación sobre el cuerpo de las mujeres,

\footnotetext{
${ }^{5}$ El término hace alusión a la idea de manchado, abigarrado, mezclado, como muchos de los ritos y danzas a los santos patronos en distintos países.
} 
particularmente de aquellas indígenas y negras, como sinónimo de subdesarrollo e ignorancia (Gargallo, 2014).

Segato (2015), en linea con la perspectiva teórico política de la colonialidad del poder, propuesta por Quijano, coincide en que el eje de la colonialidad es la invención de la raza pues marca toda la historia de América Latina. Desde allí, se producen prácticas de racialización hasta el día de hoy, en constante reproducción, considerando que la idea de raza, va más allá del fenotipo de la persona humana, y corresponde más bien a un signo:

[...] es un signo de la posición de los vencidos en la historia colonial: el racismo es epistémico, en el sentido de que las epistemes de los conquistados y colonizados son discriminadas negativamente. Racismo es eurocentrismo porque discrimina saberes y producciones, reduce civilizaciones, valores, capacidades, creaciones y creencias (Segato, 2015, p. 53).

La colonialidad surge entonces del llamado giro decolonial el cual se articula en base a tres grandes ejes, problematizar la modernidad como proyecto de desarrollo, visibilizar cómo la experiencia de dominación e imposición (a partir de la invasión a América) genera una condición de subalternidad y evidenciar el sentido etnocéntrico y eurocéntrico de las ciencias sociales, considerado como racismo epistemológico. Estas problematizaciones, resistencias y oposiciones surgen de una enérgica crítica al colonialismo, por el brutal genocidio y la dominación colonial (Dussel, 1997; Mignolo, 2005; Cusicanqui, 2010) tanto en el plano económico (apropiación de la tierra, explotación de la mano de obra de esclavos negros e indígenas y control de las finanzas), político (control de la autoridad), social (control del género y la sexualidad) como en el plano epistémico y subjetivo/personal (control del conocimiento y la subjetividad).

\section{Tercer asunto: política de representación que ha asumido el mundo letrado o intelectual}

Richard (1997) señala que algunas narrativas sobre América Latina se han convertido en un meta relato globalizador, que a través de la vía académica genera nuevas formas de dominio internacional. En una línea parecida Castro-Gómez (1998), propone deconstruir los meta discursos globalizadores sobre Latinoamérica: y coincide en que no es suficiente que las teorías postcoloniales incorporen la figura de la Otredad a su nuevo discurso anti-hegemónico para que el otro real (el sujeto concreto) formado por tramas históricas y sociales de censura y exclusión llegue a participar con voz propia en el debate metropolitano.

Para Richard (1997) existiría el monopolio del poder-de-representación según el cual "representar" significa controlar los medios discursivos que subordinan el objeto del saber a una economía conceptual que se auto declara superior, haciendo una diferencia entre hablar sobre y desde Latinoamérica. En el primer caso, la autoridad conceptual implicada en el hablar sobre Latinoamérica desde la academia internacional, se ejerce a través de una división del trabajo que, en el inconsciente de muchos de los discursos del Latinoamericanismo, suele oponer teoría y práctica: razón y materia, conocimiento y realidad, discurso y experiencia, mediación de esta cadena de oposiciones (razón, 
conocimiento, teoría, discurso, mediación) “[...] designa el poder intelectual de abstracción y simbolización que define la superioridad del Centro mientras la segunda serie (materia, realidad, práctica, experiencia, inmediatez) sitúa a América Latina en el plano de la espontaneidad de la vivencia [...]" (Richard, 1997, p. 349).

No obstante, la heterogeneidad de experiencias de vida produciría un valor popular que excede siempre la competencia académica del saber, siempre habría algo irrepresentable como el único lugar resistente de lo latinoamericano, lo que también podría implicar contra representaciones o desrepresentaciones, en tanto, “[...] prácticas recalcitrantes al orden central de clasificación académica del saber y expertas en burlar sus sistemas de valoración con imprevisibles artificios que confundan o desorganicen el control general del límite entre lo traducible y lo intraducible [...]" (Richard, 1997, p. 351).

La oposición entre representación (abstracción, teoría, discursividad) y experiencia (concreción, práctica, vivencialidad) afirma la desigualdad de poderes trazada entre, por un lado, quienes patentan los códigos de figuración teórica que dotaran a sus objetos de estudio de legitimidad académica y, por otro lado, los sujetos representados por dichos códigos, hablados por su teorización de la alteridad, pero sin mucho acceso a los beneficios institucionales de la teoría metropolitana ni derecho a ser consultados sobre la validez de las categorías que los describen e interpretan. Subvertir esta dicotomía de poder requiere producir teoría local, conocimiento situado, discurso y conciencia situacionales, que generen un desequilibrio de funciones en el interior del formato supuestamente reglado (Richard, 1997).

Otras y otros autores, dentro de los estudios poscoloniales, plantean una crítica a "[...] los mecanismos institucionales que producen representaciones del "otro u otra" y lo proyectan como una entidad susceptible de ser auscultada por los discursos etnológicos, geográficos, antropológicos, historiográficos y lingüísticos de la modernidad [...]" (Castro-Gómez, 1998, p. 4), lo que también se extiende a la situación en nuestro continente donde múltiples aproximaciones al mundo indígena, o al ciudadano latinoamericano, es exotizado o escencializado. Del mismo modo, revelan los vínculos entre imperialismo y ciencias humanas, autorizándose a sí mismas a construir discursivamente una imagen de las "[...] culturas no metropolitanas, especialmente de aquellas que se encuentran bajo su control territorial [...]” (Castro-Gómez, 1998, p. 4). Lo mismo ocurre en América Latina, por tanto, es claro que toda imagen de América Latina siempre está cruzada por el poder que la patrocina.

\section{Cuarto asunto: colonialidad interna}

Para finalizar nos parece muy importante señalar lo que Cusicanqui (2010) subraya y critica respecto de algunas narrativas anticoloniales, incluso de autores muy renombrados del grupo colonialidad modernidad, entre los cuales se encuentra Walter Mignolo y el mismo Aníbal Quijano que dan cuenta de procesos de colonialidad interna. Para la autora, existe una autoría indígena silenciada por la academia, que ha hecho suyos una serie de reflexiones que ya se venían desarrollando, por ejemplo, en Bolivia. Una de las principales se relaciona con la idea de descolonización, tema que introduce en su libro "Oprimidos, pero no vencidos" (Cusicanqui, 1984), mucho antes de las elaboraciones de Quijano (1998) sobre el mismo asunto. A partir de esto, en el año 
2010, en su libro "Chìxinakax Utxima. Una reflexión sobre prácticas y discursos descolonizadores", Silvia Rivera Cusicanqui plantea tres críticas importantes para reflexionar sobre la colonialidad interna, la primera, es la necesaria transformación de las formas de comprender la realidad global y local de quien narra, esto implica una "[...] radical y profunda descolonización en sus estructuras políticas, económicas y sobre todo mentales, es decir en sus modos de concebir el mundo [...]” (Cusicanqui, 2010, p.56). La segunda critica, hace referencia a las citaciones de autores señalando que "[...] a través del juego de quien cita a quién, se estructuran jerarquías y acabamos teniendo que comer y regurgitando, el pensamiento descolonizador que las poblaciones e intelectuales indígenas de Bolivia, Perú y Ecuador habíamos producido independientemente [...]” (Cusicanqui, 2010, p. 66). Finalmente, la tercera crítica que realiza la autora, hace alusión a la importancia de reconocer la colonialidad interna que nos recorre, que cruza la academia y también a aquella intelectualidad que aboga sobre América Latina, pues no es inmune a la matriz de poder y a los ejercicios "[...] de cooptación y mimesis, incorporación selectiva de ideas, selección certificadora de cuales son más válidas para alimentar [...]" (Cusicanqui, 2010, p. 68), en este caso, a su institucionalidad disciplinar.

\section{América Latina y SUS contribuciones a las terapias ocupacionales del sur}

En los años de 1970 dentro de las terapias ocupacionales que, aproximadamente una década antes empiezan a surgir en América Latina, algunas voces divergentes se hacen visibles y comienzan a resonar, voces que hacen una crítica a los discursos totalizantes y reivindican múltiples voces desde las periferias o bordes del tradicional ejercicio teórico y práctico de la profesión/disciplina (Oliver, 1990; Barros, 1991; Guajardo, 1992; Lopes, 1997; Paganizzi, 1997; Barros et al., 1999; Oliver \& Nicácio, 1999; Malfitano, 2005). Progresivamente se crean las causas y condiciones que tensionan el modus operandi de la profesión a partir de la pluralidad de prácticas locales y concepciones de mundo que terminan por sedimentar en lógicas que reconocen la pluriversalidad ${ }^{6}$ (Mignolo, 2007; Santos, 2018) por sobre las comprensiones universalizantes de la terapia ocupacional. En un cierto sentido, se reconoce un legado indiscutible, un patrimonio que se dispersa con distintas tonalidades en la región (Díaz, 2018) y que, se encarna en estas comunidades hasta el día de hoy, en otro sentido, se advierte un potencial creador que emerge de las formas de vida en América Latina, cruzados inevitablemente por las huellas coloniales, lo que genera la necesidad de una praxis situada. Surge así, un discurso desde América Latina, no exento de heterogeneidades contradictorias, una manera de pensar la profesión, ontológica y epistemológicamente (Oliver, 1990; Lopes, 1997; Guajardo \& Galheigo, 2015; Lopes \& Malfitano, 2016), a partir de los contextos históricos específicos de la región y de cada país, ya no como un producto de imitación del centro hacia la periferia sino como una posibilidad de reconocer los contextos sociales, políticos, económicos que le van dando otras formas. No obstante, así como esta nueva posicionalidad tiene una fuerte potencialidad, también no ha estado exenta como toda práctica social, de las huellas de la colonialidad que en este texto hemos tratado de resumir en cuatro asuntos.

${ }^{6}$ Entendido como nudos de una red genealógica desde donde se produce un "[...] punto de despegue y apertura que reintroduce lenguas, memorias, economías, organizaciones sociales, subjetividades, esplendores y miserias de los legados imperiales [...]” (Mignolo, 2007, p. 45). 
$\mathrm{Al}$ respecto, la primera cuestión que nos parece importante señalar a partir de lo que proponen estas diversas narrativas en torno a América Latina, es la de concebir cómo sus historias fueron creando y re-creando las praxis de las terapias ocupacionales de una manera singular en nuestro continente como una acción en el mundo, siempre historizada, localizada y situada (Guajardo, 2016). En cada país, su configuración tuvo directa relación con los diversos proyectos históricos que cada Estado Nación tuvo que enfrentar tras la implantación de un proyecto moderno que, del mismo modo, se desplegó en cada territorio de manera singular. Esta lectura, nos permite liberar las diferencias y no prolongar esta vieja pero siempre presente búsqueda de un origen o de fundamentos universalistas, que nos confieran una identidad, del latín identitas, lo mismo. Hay muchos comienzos y re-comienzos, giros, desvíos, por tanto, nunca una historia lineal, ni única, como bien lo señala Adichie (2009) al enfatizar en los peligros de una historia única. Las prácticas de las terapias ocupacionales como parte de las prácticas de la vida en este continente, son escurridizas e imposibles de delimitar y contener en palabras, términos o categorías, siempre rebasan el discurso académico que buscando representarlas no logra siquiera identificarlas en su vasta diversidad. Por lo tanto, en relación al primer asunto, la heterogeneidad contradictoria de América Latina, está presente también en la misma terapia ocupacional caracterizada por una amplia heterogeneidad y múltiples historias que componen las terapias ocupacionales en nuestro continente.

Con respecto al segundo asunto, la colonialidad como legado trans-generacional de la colonización, es difícil pensar que la formación académica y sus prácticas hayan resultado ilesas de ese acontecer histórico que hasta el día de hoy pervive. Esa matriz de poder que instauró y sigue instaurando nuevas formas de dominio en todas direcciones, sea como colonización, neo-colonización o globalización en el plano político, económico, social, epistémico y subjetivo, nos interpela a escudriñar los modos en que se encarnó en el discurso, en la academia y en sus prácticas. Activar una crítica que no es exterior a sí misma, es una condición necesaria más no suficiente, pues se requieren las propuestas para frenar ese proyecto siempre insaciable y en gran medida devastador.

La colonialidad operó de diversas formas, en el mundo, en el continente, y dentro de cada continente y país. Así, la implantación del capitalismo, por ejemplo, en América se dio fuertemente por la explotación y uso de diversas formas de esclavitud, los procesos de modernización con la introducción y uso de recursos y tecnologías diferenciados, el modernismo y sus tendencias artísticas con más o menor apertura y las universidades adquirieron la tarea del progreso y la función civilizatoria con mayor o menor confianza y anhelo. Entonces, es fácil comprender porque la terapia ocupacional se desarrolló y lo sigue haciendo, de maneras tan particulares en América del Sur, América Central, Caribe y parte de América del Norte. De tal modo, pensar la colonialidad como legado transgeneracional de la colonización en la terapia ocupacional implica reconocer la fuerte influencia de los condicionantes económicos y políticos en el desarrollo de la profesión y de cómo históricamente, pudiéramos haber estado al servicio del sistema moderno/colonial/capitalista/patriarcal en nuestras prácticas profesionales.

Con relación a las politicas de representación que ha asumido el mundo letrado o intelectual, en la terapia ocupacional es posible identificar los riesgos de monopolizar el saber y la producción de conocimientos en la región, por la fuerte influencia anglosajona, cartesiana, sea como saber médico o saber cientificista. Por cierto, es sabido que algunos 
colectivos de terapeutas ocupacionales mostraron su distanciamiento e indisposición a ser representados por ese único discurso o tener que representar a los sujetos de su intervención bajo una única ontología, epistemología y metodología ¿cómo se ha configurado la relación entre la terapia ocupacional y su particular alteridad? ¿a quienes definió como sujetos de estudio u objetos de intervención? ¿de qué modo se problematizaron las relaciones desiguales de género, de clase y étnico raciales? ¿en qué medida nombró y con ello pre-figuró, sea como enferma(o), paciente o caso, a sus sujetos de intervención o investigación? ¿de qué forma esto permitió incorporar otros términos como usuarias(os), consultantes, clientes, grupos vulnerables? En otras palabras, estos distintos medios discursivos ¿modificaron las relaciones de subordinación $\mathrm{o}$ subalternización dentro del ejercicio profesional? Se requiere entonces la interrogación permanente de su posicionalidad para no asumirla de manera mitificada o escencializada pues aun cambiando los conceptos utilizados, paradojalmente, puede reproducir el mismo orden y una figura de la alteridad como mera representación y no como coexistencia.

De este modo, las politicas de representación que ha asumido el mundo letrado o intelectual se reflejan en la terapia ocupacional por la reproducción de saber médico o saber cientificista sin considerar otro tipo de saberes, generando una relación de subalternización de saberes o de mera representación, por ejemplo, cuando la o el terapeuta ocupacional actúa y trabaja sin cuestionar las explicaciones médicas frente a situaciones que se relacionan con problemas estructurales de la sociedad.

Finalmente, la idea de colonialidad interna nos parece el punto de partida, esto es interpelar a quienes narramos, actuamos o intentamos producir saberes con las realidades de esta región, pues necesariamente asumimos un lugar de enunciación que no es exterior ni ajeno a aquello que se narra. Implica una posición reflexiva sobre nuestras prácticas y las divisiones y asimetrías de poder, en todos los escenarios del quehacer profesional/disciplinar. Asumir entonces una narrativa desde Latinoamérica, implica asumir al mismo tiempo, una lógica que no homogenice a los sujetos, producciones y prácticas de este continente, por el riesgo de escencializar, victimizar o atribuir un grado de heroísmo que impide el encuentro con el otro/otra, dejando a unos en la posición del saber del intelectual/profesional y al otro en el lugar fijo de subalterno.

Desde el panóptico académico y particularmente desde el panóptico logocentrista de la terapia ocupacional es fundamental no apresar con nuestros conceptos o terminologías aquello que es imposible uniformar y que difícilmente puede explicarse exclusivamente desde el ámbito técnico, cuestión que ocurre cuando se utiliza la terminología uniforme de la Asociación Americana de Terapia Ocupacional para evaluar y describir las situaciones que afectan a grupos en contextos de desigualdad estructural en Latino América. Es importante tomar las precauciones necesarias cuando queremos crear nuevos conceptos para nombrar algo, sea para movilizar alguna transformación, tensión o ruptura con otras categorías o, sea para desmarcarnos de una posición que es insuficiente para explicar y comprender un campo especifico de práctica, teniendo cuidado con el uso de categorías que intentando ampliar pueden más bien recortar, por ejemplo, al agregar el término "ocupacional" a términos cuya procedencia y sentido exceden las fronteras de una profesión o disciplina, es el caso del concepto de justicia social y su contracción mediante el uso del concepto de "justicia ocupacional”. En otras palabras, la colonialidad interna también se puede expresar en la búsqueda de legitimar 
la terapia ocupacional y sus fronteras, marcando el perímetro de ésta con la marca "ocupacional" a categorías y conceptos absolutamente transdisciplinares que tienen un fuerte acervo histórico y que dialogan con otras historias en la terapia ocupacional latinoamericana.

\section{Discusión Final}

Nos parece relevante considerar las narrativas sobre América Latina para pensar la actuación de la terapia ocupacional en nuestro continente. Así, los cuatro asuntos abordados tuvieron como objetivo ofrecer aportes al debate sobre las Terapias Ocupacionales del Sur, pues pensar sobre la idea de América Latina, nos permite identificar las maneras en que se transformó el sentido de la profesión y se siguen produciendo nuevos sentidos y prácticas en respuesta a los contextos históricos y temporalidades en este continente. La intensificación de las prácticas escriturarias, a través de artículos, libros y otros medios de difusión, con un fuerte sentido latinoamericano son un reflejo de ello y de su voluntad de no "[...] dejar de leer, dialogar y participar en los movimientos globales de la profesión [...]” (Botinelli et al., 2016, p. 97).

Sabemos que se trata de una reflexión parcial sobre el tema y queda mucho por profundizar, nuestro objetivo no era cubrir todo el tema, ni proponer una teoría sobre la conceptualización de América Latina para las terapias ocupacionales desarrolladas en el sur, más bien, fue plantear algunas ideas que nos permitieran establecer un dialogo inicial entre el campo disciplinar y las teorías sociales que discuten lo latinoamericano y las diversas formas de colonialidad en nuestro continente, centrándose en su aplicación a la profesión. De manera específica, las narrativas sobre la "idea de América Latina", nos permiten problematizar nuestros análisis de los sujetos y prácticas - tanto en el campo interventivo, investigativo o de cualquier índole- y analizar las políticas de representación o los modos en que hemos y seguimos produciendo la alteridad en el campo de las terapias ocupacionales. En el caso de nuestro quehacer profesional, esa alteridad se vincula muy directamente con sujetos o comunidades en situación de discapacidad, niñas(os) en situación de calle, migradas(os) latinoamericanas(os), entre otros, y cómo nosotras(os) mismas(os) estamos siendo producidas(os) en esa relación ¿mera representación o coexistencia?

Reflexionar a partir de estos cuatro asuntos, nos interpela a comprender cómo se han producido determinados modos de sentir, pensar, ser y hacer terapia ocupacional en América Latina. Esperamos que la conceptualización de América Latina pueda estar presente en las terapias ocupacionales que hacemos influyendo en sus prácticas, dirigidas a nuestras realidades locales, donde el dialogo entre la acción y el desarrollo teórico es fundamental y debe ser asumido por todas y todos, especialmente por quienes nos situamos en el campo académico. Estos diálogos nos invitan a reflexionar también en los modos de vida de las personas y las comunidades con que estamos trabajando a partir de una narrativa desde el sur y desde Latinoamérica, lo que debería movilizar a las terapias ocupacionales del continente para construir proyectos colectivos contrarios a las lógicas capitalistas, patriarcales y racistas, consustanciales de la colonialidad en América 
Latina. De allí, que podemos decir que un pensamiento al sur ${ }^{7}$, es la posibilidad de mantener abierto los diálogos e intercambios, conectando las diferentes experiencias de los países latinoamericanos, más allá de un solo marco instituido para pensar la terapia ocupacional.

\section{Referencias}

Adichie, C. N. (2009). O perigo da história única. Recuperado el 31 de marzo de 2014, de http://www.ted.com/talks/lang/pt/chimamanda_adichie_the_danger_of_a_single_story.html

Anzaldúa, G. (1987). Borderland/ La frontera: The new Mestiza. San Francisco: Aunt Lute Books.

Barros, D. D. (1991). Habilitar - reabilitar: o rei esta nú? Revista de Terapia Ocupacional da Universidade de São Paulo, 2(2/3), 100-104.

Barros, D. D., Ghirardi, M. I. G., \& Lopes, R. E. (1999). Terapia ocupacional e sociedade. Revista de Terapia Ocupacional da Universidade de São Paulo, 10(2/3), 69-74.

Benhabib, S. (2006). El ser y el otro en la ética contemporánea. Barcelona: Editorial Gedisa.

Borda, O. F. (2009). Una Sociología Sentipensate para América Latina. Bogotá: CLACSO/ Siglo del Hombre Editores.

Botinelli, M., Nabergoi, M., Albino, A., \& Benassi, J. (2016). ¿Por qué pensar epistemología en Terapia Ocupacional? In S. S. Algado, A. G. Córdoba, F.C. Oliver, S.M. Galheigo \& S. García-Ruiz (Eds.), Terapias Ocupacionales del Sur (pp. 97-112). Santiago: Editorial USACH.

Castro-Gómez, S. (1998). Latinoamericanismo, modernidad, globalización. Prolegómenos a una crítica poscolonial de la razón. In S. Castro-Gomez \& E. Mendieta (Eds.), Teorías sin disciplina (pp. 118150). México: Editorial Miguel Ángel Porrúa.

Cottet, P. (2015). Emplazamientos contemporáneos al quehacer de las ciencias sociales. In I Congreso Latinoamericano de Teoría Social. Instituto de Investigaciones Gino Germani. Facultad de Ciencias Sociales, Universidad de Buenos Aires, Buenos Aires, Argentina.

Cusicanqui, S. R. (1984). Oprimidos, pero no vencidos: luchas del campesinado aymara y qhechwa 19001980. La Paz: Hisbol.

Cusicanqui, S.R. (2010). Chixinakax Utxima: una reflexión sobre prácticas y discursos descolonizadores. Buenos Aires: Tinta limón.

Díaz, M. (2018). Terapias ocupacionales: migraciones de saberes y prácticas en Latinoamérica. Revista Ocupación Humana, 18(1), 21-33.

Duarte, C., Fernández, A., Cruz, J., \& García, S. (2016). Precursores de la terapia ocupacional en Colombia: sujetos, instituciones, oficios. Revista Ocupación Humana, 16(2), 93-109.

Dussel, E. (1997). Introducción a la filosofia de la Liberación: ensayos preliminares y bibliografia. Bogotá: Nueva América.

Fernandes, F. (1973). Capitalismo dependente e classes sociais na América Latina. Rio de janeiro: Zahar.

Galheigo, S. M. (2003). O cotidiano na terapia ocupacional: cultura, subjetividade e contexto históricosocial. Revista de Terapia Ocupacional da Universidade de São Paulo, 14(3), 104-109.

Garcia, F. (2012). Hacia una Razón Situada. Santiago: Editorial LOM.

Gargallo, F. (2014). Feminismos desde Abya Yala: ideas y proposiciones de las mujeres de 607 pueblos en nuestra América. Ciudad de México: Editorial Corte y Confección. Recuperado el 10 de junio de 2014, de http://francescagargallo.wordpress.com

\footnotetext{
${ }^{7}$ Se puede localizar en distintos puntos geográficos que comparten el mismo lugar de enunciación, como América Central, África, Asia y Europa. Así mismo, habitar el sur geográfico, no es sinónimo de prácticas al sur de la terapia ocupacional.
} 
Guajardo, A. (1992). Una aproximación al quehacer de terapia ocupacional en la persona torturada. In Anais do 2o Seminario de la Región del Maule (pp. 55- 59). Chile: CINTRAS.

Guajardo, A. G. (2013). Lecturas y relatos históricos de la Terapia Ocupacional en Suramérica: una perspectiva de reflexión crítica. Revista Ocupación Humana, 16(2), 110-117.

http://dx.doi.org/10.25214/25907816.141.

Guajardo, A. G., \& Galheigo, S. M. (2015). Reflexiones críticas acerca de los derechos humanos: contribuciones desde la terapia ocupacional Latinoamericana. WFOT Bulletin, 71(2), 73-80.

Guajardo, A. (2016). Construcción de identidades, epistemes y prácticas en Terapia Ocupacional en Latinoamérica. In S. Simó, A. Guajardo, F. Oliver, S. Galheigo, \& S. García (Eds.), Terapias Ocupacionales desde el Sur. Derechos Humanos, ciudadanía y participación (pp. 41-62). Santiago de Chile: Editorial Usach

Herrera, M., \& Valderrama, C. (2013). Gubernamentalidad y biopolítica: una aproximación con los saberes y prácticas históricas de la terapia ocupacional en Chile. Revista Chilena de Terapia Ocupacional, 13(2), 79-92.

Lopes, R. E. (1997). A direção que construímos: algumas reflexões sobre a formação do terapeuta ocupacional. Revista de Terapia Ocupacional da Universidade de São Paulo, 4(7), 27-35.

Lopes, R. E., \& Malfitano, A. P. S. (2016). Terapia ocupacional social: desenhos teóricos e contornos práticos. São Carlos: EdUFSCar.

Malfitano, A. P. S. (2005). Campos e núcleos de intervenção na terapia ocupacional social. Revista de Terapia Ocupacional da Universidade de São Paulo, 16(1), 1-8. http://dx.doi.org/10.11606/issn.2238-6149.v16i1p1-8.

Mignolo, W. (2005). La idea de América Latina: la herida colonial y la opción decolonial. Barcelona: Editorial Gedisa.

Mignolo, W. (2007). El pensamiento decolonial: desprendimiento y apertura. In S. C. Gomez \& R. Grosfoguel (Eds.), El giro decolonial: refl exiones para una diversidad epistémica más allá del capitalismo global (pp. 25-46). Bogotá: CLACSO/Siglo del Hombre Editores.

Moreiras, A. (1996). Elementos de articulación teórica para el subalternismo latinoamericano: cándido y Borges. Revista Iberoamericana, 176-177, 875-891.

Oliver, F. (1990). A atenção à saúde da pessoa portadora de deficiência no município de São Paulo: uma questão de cidadania (Dissertaçáo de mestrado). Universidade de São Paulo, São Paulo.

Oliver, F. C., \& Nicácio, F. (1999). Da instituição asilar ao território: caminhos para a produção de sentido nas intervenções em saúde. Revista de Terapia Ocupacional da Universidade de São Paulo, $10(2 / 3), 67-70$.

Paganizzi, L. (1997). Actividad: lenguaje particular. Buenos Aires: Talleres gráficos Edigraf S.A.

Quijano, A. (1998). La colonialidad del poder y la experiencia cultural latinoamericana. In R. BriceñoLeón \& H. R. Sonntag (Eds.), Pueblo, Época y desarrollo: la sociología de América Latina (pp. $117-$ 142). Caracas: Editorial Nueva Sociedad.

Richard, N. (1997). Intersectando Latinoamérica con el Latinoamericanismo saberes académicos, práctica teórica y crítica cultural. Revista Iberoamericana, LXIII(180), 345-361.

Santos, B. S. S. (2018).Ecología de Saberes. In B. S. Santos, M. P. Meneses, J. A. Nunes, C. L. Añón, A. A. Bonet \& N. L. Gomes (Eds.), Construyendo las Epistemologías del Sur: para un pensamiento alternativo de alternativas (pp. 229-266). Buenos Aires: CLACSO/Siglo del Hombre Editores.

Segato, R. (2015). La crítica de la colonialidad en ocho ensayos: Y una antropología por demanda. Buenos Aires: Prometeo.

Testa, D. E., Narváez, S., Mariscal, C., Sartirana, A. G., Caillet-Bois, C., \& Albino, A. (2016). Pluralidades y desafíos en la construcción del conocimiento. Revista Argentina de Terapia Ocupacional, 2(1), 1-2. 
Zorzoli, M., Chaura, L., \& Paganizzi, L. (2014). Contextos habitados por sujetos, desafíos de la terapia ocupacional: diversidad, organización y movimientos actuales. In V. Santos \& A. D. Gallassi (Orgs.), Cuestiones contemporáneas de terapia ocupacional en América del Sur (pp. 19-34). Curitiba: CRV.

\section{Contribución de las Autores}

Mónica Matilde Díaz Leiva es la autora de la tesis de doctorado en desarrollo, sobre la temática de este artículo, con la supervisión de Ana Paula Serrata Malfitano. Mónica Matilde Díaz Leiva es responsable por el desarrollo central del texto y Ana Paula Serrata Malfitano de su supervisión y revisión. Todas las autoras aprueban la version final del texto.

\section{Fuente de Financiamiento}

El presente trabajo se realizó con el apoyo de la Coordenação de Aperfeiçoamento de Pessoal de Nível Superior - Brasil (CAPES) - Código de Financiamiento 001.

\section{Autor para la correspondencia}

Mónica Matilde Díaz Leiva

e-mail monica.diaz.1@usach.cl

\section{Section editor}

Profa Dra. Sandra Maria Galheigo 